\title{
Dimensional Reduction Applied to QCD at Higher Orders
}

\author{
Robert Harlander ${ }^{1}$, Philipp Kant ${ }^{2}$, Luminita Mihaila ${ }^{2}$, Matthias Steinhauser ${ }^{2}$ * \\ 1- Fachbereich C - Bergische Universität Wuppertal \\ 42097 Wuppertal - Germany \\ 2- Theoretische Teilchenphysik - Universität Karlsruhe \\ 76128 Karlsruhe - Germany
}

\begin{abstract}
Recent developments in higher order calculations within the framework of Dimensional Reduction, the preferred regularization scheme for supersymmetric theories, are reported on. Special emphasis is put on the treatment of evanescent couplings, the equivalence to Dimensional Regularization, and the evaluation of $\alpha_{s}\left(M_{\mathrm{GUT}}\right)$ from $\alpha_{s}\left(M_{\mathrm{Z}}\right)$.
\end{abstract}

\section{Dimensional Reduction}

Dimensional Regularization (DREG) [2] has proven extremely successful for the evaluation of higher order corrections in quantum field theory, mostly because it preserves gauge invariance and thus does not interfere with the renormalizability of the Standard Model or QCD. Many techniques for evaluating Feynman diagrams have been developed within the framework of DREG, so perturbation theory heavily relies upon the validity of this regularization method.

Applied to SUSY theories, however, one faces the problem of explicit SUSY breaking by the need to assign different numbers of degrees of freedom to spin- 1 and spin- $1 / 2$ fields. A manifestation of this SUSY breaking is that SUSY relations of couplings no longer hold at higher orders. For example, while SUSY requires equality for the quark-quark-gluon and the squark-quark-gluino couplings $g$ and $\hat{g}$ at all energy scales, one finds that their renormalization constants differ. In fact, it is $Z_{g}=\left(1+\delta_{\hat{g}}\right) Z_{\hat{g}}$, and thus

$$
\hat{g}=\left(1+\delta_{\hat{g}}\right) g,
$$

where $\delta_{\hat{g}}=\alpha_{s} /(3 \pi)[3]$. In effect, the number of renormalization constants in SUSY becomes rather large when calculations are done in DREG.

As a way out, it was suggested to use Dimensional Reduction (DRED) as a regularization procedure for SUSY theories [4]. Formally, this means that space-time is compactified to $D=4-2 \epsilon$ dimensions $(\epsilon>0)$, while the vector fields are kept four-dimensional. As an example, consider the electron-photon vertex, which in DRED becomes

$$
\bar{\psi} \gamma_{\mu} \psi A^{\mu}=\bar{\psi} \gamma_{\mu} \psi \hat{A}^{\mu}+\bar{\psi} \gamma_{\mu} \psi \tilde{A}^{\mu}=\bar{\psi} \hat{\gamma}_{\mu} \psi \hat{A}^{\mu}+\bar{\psi} \tilde{\gamma}_{\mu} \psi \tilde{A}^{\mu}
$$

where $\hat{A}^{\mu}$ and $\tilde{A}^{\mu}$ denote the $D$ and the $2 \epsilon$-dimensional component of the vector field $A^{\mu}$. $\tilde{A}^{\mu}$ is also called the $\epsilon$-scalar. Traces over the $D$ - and $2 \epsilon$-dimensional $\gamma$-matrices can be evaluated using

$$
\left\{\gamma^{\mu}, \gamma^{\nu}\right\}=2 g_{\mu \nu} \quad \text { and } \quad\left\{\hat{\gamma}^{\mu}, \tilde{\gamma}^{\nu}\right\}=0 .
$$

Thus, perturbative calculations in DRED require to introduce additional fields ( $\epsilon$-scalars) and an extra set of $\gamma$-matrices. Once the algebraic part of the evaluation of a Feynman amplitude is done, the tools developped for DREG can be applied without further modification.

\footnotetext{
*Work supported by the DFG through SFB/TR 9 and HA 2990/3-1.
} 


\section{Evanescent couplings}

In a SUSY theory, the relation $A_{\mu}=\hat{A}_{\mu}+\tilde{A}_{\mu}$ is essential: it is $A_{\mu}$ that is part of a supermultiplet, while $\hat{A}_{\mu}$ and $\tilde{A}_{\mu}$ are introduced for purely technical reasons. Therefore, this relation must hold also at higher orders of perturbation theory.

This is not necessarily the case in a non-SUSY theory. Since $\tilde{A}_{\mu}$ transforms like a scalar under gauge transformations (thus the name " $\epsilon$-scalar"), there is no symmetry to ensure that the $\tilde{A}_{\mu}$-couplings renormalize in the same way as the corresponding $\hat{A}_{\mu}$-couplings. Thus, when applying DRED to QCD, we have to introduce two different couplings for the quarkgluon vertex, for example:

$$
g_{s} A_{\mu} \bar{\psi} \gamma^{\mu} \psi \rightarrow \hat{g}_{s} \hat{A}_{\mu} \bar{\psi} \hat{\gamma}^{\mu} \psi+\tilde{g}_{s} \tilde{A}_{\mu} \bar{\psi} \tilde{\gamma}^{\mu} \psi .
$$

In order to be consistent with our journal papers $[5,6,7]^{\mathrm{a}}$ let us define

$$
\alpha_{s}=\frac{\hat{g}_{s}^{2}}{4 \pi}, \quad \alpha_{e}=\frac{\tilde{g}_{s}^{2}}{4 \pi},
$$

where $\alpha_{e}$ is called "evanescent coupling". Only at tree-level can one require that $\alpha_{s}=\alpha_{e}$. Higher orders lead to an energy dependece of the (minimally subtracted) couplings, governed by the RGEs ${ }^{\mathrm{b}}$

$$
\mu^{2} \frac{\mathrm{d}}{\mathrm{d} \mu^{2}} \alpha_{s}=\beta_{s}^{\overline{\mathrm{DR}}}\left(\alpha_{s}, \alpha_{e}\right), \quad \mu^{2} \frac{\mathrm{d}}{\mathrm{d} \mu^{2}} \alpha_{e}=\beta_{e}\left(\alpha_{s}, \alpha_{e}\right)
$$

The $\beta$-functions have been evaluated in Ref. [5] through three loops, and $\beta_{s}^{\overline{\mathrm{DR}}}$ is even known to four-loop order [6]. Indeed it turns out that $\beta_{s}^{\overline{\mathrm{DR}}} \neq \beta_{e}$ in standard QCD already at one-loop level. The condition $\alpha_{s}=\alpha_{e}$ can therefore be implemented only at one particular value of $\mu^{2}$.

If $\overline{\mathrm{DR}}$ (i.e., DRED with minimal subtraction) is to be a viable renormalization scheme, then one should be able to transform physical results from one scheme into the other by finite shifts of the renormalized parameters. This property has been confirmed several times $[8,5,6]$. The proper conversion relation for the strong coupling between the $\overline{\mathrm{MS}}$ and the $\overline{\mathrm{DR}}$ scheme in $n_{f}$-flavor standard QCD is given at two-loop level by [5]

$$
\bar{\alpha}_{s}=\alpha_{s}\left[1-\frac{\alpha_{s}}{4 \pi}-\frac{5}{4}\left(\frac{\alpha_{s}}{\pi}\right)^{2}+\frac{\alpha_{s} \alpha_{e}}{12 \pi^{2}} n_{f}+\ldots\right],
$$

where $\bar{\alpha}_{s}$ denotes the strong coupling in the $\overline{\mathrm{MS}}$ scheme. Three-loop corrections to this relation are known as well [6].

When evaluating physical observables in DREG, the result depends only on $\bar{\alpha}_{s}$, while it depends on both $\alpha_{s}$ and $\alpha_{e}$ in DRED. This ambiguity should be viewed as a freedom of the renormalization scheme: any choice of $\alpha_{e}$ determines the value of $\alpha_{s}$ by comparison to the experimental value of the physical observable at one particular scale $\mu_{0}$. At any other scale $\mu, \alpha_{s}$ and $\alpha_{e}$ are determined by the RGEs Eq. (6).

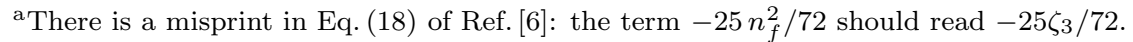

${ }^{\mathrm{b}}$ In fact, there are several evanescent couplings in $\mathrm{QCD}$; however, for the sake of the argument, it is sufficient to consider only $\alpha_{e}$ here.
} 
Also, there is a unique relation between the perturbative coefficients of the $\overline{\mathrm{DR}}$ and the $\overline{\mathrm{MS}}$ expression of the physical observable, to be called $R$ and $\bar{R}$ in what follows. For example, assume that

$$
R\left(\alpha_{s}, \alpha_{e}\right)=\sum_{i, j \geq 0}\left(\frac{\alpha_{s}}{\pi}\right)^{i}\left(\frac{\alpha_{e}}{\pi}\right)^{j} r_{i j}, \quad \bar{R}\left(\bar{\alpha}_{s}\right)=\sum_{i \geq 0}\left(\frac{\bar{\alpha}_{s}}{\pi}\right)^{i} \bar{r}_{i} .
$$

Then, inserting Eq. (7) into Eq. (8) and requiring equality, one derives the relations

$$
\begin{array}{r}
r_{00}=\bar{r}_{0}, \quad r_{10}=\bar{r}_{1}, \quad r_{01}=0, \quad r_{20}=\bar{r}_{2}-\frac{\bar{r}_{1}}{4}, \quad r_{02}=0, \quad r_{11}=0, \\
r_{30}=\bar{r}_{3}-\frac{\bar{r}_{2}}{2}-\frac{5}{4} \bar{r}_{1}, \quad r_{21}=\frac{n_{f}}{12} \bar{r}_{1}, \quad r_{12}=0, \quad r_{03}=0, \quad \text { etc. }
\end{array}
$$

\section{Relation of $\alpha_{s}$ and $\alpha_{e}$ by Supersymmetry}

Supersymmetry is a concept that provides solutions to some of the most pressing questions left open by the Standard Model. As already mentioned above, in a SUSY theory it is required that $\alpha_{s}=\alpha_{e}$ at all energy scales, and thus $\beta_{s}=\beta_{e}$. We can use the QCD results of Ref. [5] to test the consistency of DRED and SUSY for a SUSY Yang Mills theory at three-loop level, simply by choosing the color factors appropriately. Indeed, we find that $\beta_{s}=\beta_{e}$ through three loops in a SUSY Yang Mills theory. For a check of this relation within SUSY-QCD, one needs to include chiral fields in the fundamental representation of the gauge group, or in other words, quarks and squarks. This is work in progress.

If indeed the QCD that we observe is the low energy limit of a softly broken SUSY-QCD theory, then the freedom of choosing $\alpha_{e}$ is lost, because within this SUSY theory, we require $\alpha_{e}^{(\text {full })}=\alpha_{s}^{(\text {full })}$ at all scales. The couplings in QCD are related to those in SUSY-QCD by matching relations:

$$
\alpha_{s}(\mu)=\zeta_{s} \alpha_{s}^{(\text {full })}(\mu), \quad \alpha_{e}(\mu)=\zeta_{e} \alpha_{e}^{(\text {full })}(\mu),
$$

where $\zeta_{s}$ and $\zeta_{e}$ are functions of $\alpha_{s}^{(\text {full })}$, the SUSY particle masses, and the "matching scale" $\mu$ (if $\alpha_{s}$ and $\alpha_{e}$ are the couplings in five-flavor QCD, then $\zeta_{s}$ and $\zeta_{e}$ depend also on the top quark mass). Note that since the dependence of $\zeta_{s, e}$ on the matching scale $\mu$ is logarithmic, one should apply Eq. (10) at a scale not too much different from the SUSY particle masses. Also, if these masses are spread over a large range, matching better be done in several steps.

$\zeta_{s}$ and $\zeta_{e}$ can be evaluated perturbatively. The two-loop expression for $\zeta_{s}$ has been calculated in Ref. [9], while for $\zeta_{e}$ only the one-loop term is known [7].

Assume now for the sake of the argument that all SUSY-QCD particle masses are identical, say $m_{\tilde{q}}=m_{\tilde{g}}=\tilde{M} \sim 1 \mathrm{TeV}$. If $\bar{\alpha}_{s}\left(M_{Z}\right)$ in QCD is given by experiment, then the SUSY coupling, for example at the GUT scale $\mu_{\mathrm{GUT}}$, can be determined by the following scheme:

$$
\bar{\alpha}_{s}\left(M_{Z}\right) \stackrel{(i)}{\longrightarrow} \bar{\alpha}_{s}\left(\mu_{\mathrm{dec}}\right) \stackrel{(i i i)}{\longleftarrow}\left\{\begin{array}{l}
\alpha_{s}\left(\mu_{\mathrm{dec}}\right) \\
\alpha_{e}\left(\mu_{\mathrm{dec}}\right)
\end{array}\right\} \stackrel{(i i)}{\longleftarrow} \alpha_{s}^{(\mathrm{full})}\left(\mu_{\mathrm{dec}}\right) \stackrel{(i v)}{\longrightarrow} \alpha_{s}^{(\mathrm{full})}\left(\mu_{\mathrm{GUT}}\right) .
$$

If the evolution is to be consistent through $n$-loop order, then steps $(i)$ and $(i v)$ need to be done through $n$ loops, while steps $(i i)$ and $(i i i)$ are only required through $(n-1)$ loops. Here, it is understood that one starts with a trial value $\alpha_{0}$ for $\alpha_{s}^{(\text {full })}\left(\mu_{\mathrm{dec}}\right)$, evaluates steps $(i i)$ and 
(iii), and compares the value for $\bar{\alpha}_{s}\left(\mu_{\mathrm{dec}}\right)$ obtained in this way with the one obtained from step $(i)$. If it agrees, one performs step $(i v)$ with $\alpha_{s}^{(\text {full) }}\left(\mu_{\mathrm{dec}}\right)=\alpha_{0}$, otherwise, one starts again with a different value for $\alpha_{0}$.

An alternative way to proceed was applied in Ref. [7]. There, the relation between $\alpha_{s}\left(\mu_{\mathrm{dec}}\right)$ and $\alpha_{e}\left(\mu_{\mathrm{dec}}\right)$ was perturbatively expanded such that $\alpha_{s}\left(\mu_{\mathrm{GUT}}\right)$ could be directly evaluated from $\bar{\alpha}_{s}\left(M_{Z}\right)$ without the need for an iterative procedure. The difference between these two approaches is formally of higher orders in $\alpha_{s}$, but is expected to grow as the decoupling scale moves away from the SUSY masses $\tilde{M}$. At three-loop level, the two approaches are consistent with each other within each others uncertainty (derived from the experimental error on $\alpha_{s}\left(M_{Z}\right)$ [11]) over a large range of the decoupling scale. Figure 1 shows the result [7], demonstrating the numerical importance of the three-loop effects, in particular if decoupling is done at other scales than $\tilde{M}$ (quite often one finds $\mu_{\text {dec }}=M_{Z}$, for example [10]).

\section{Conclusions}



Figure 1: $\alpha_{s}$ at $\mu_{\mathrm{GUT}} \equiv 10^{16} \mathrm{GeV}$ derived from $\alpha_{s}\left(M_{Z}\right)$ in 1-, 2-, and 3-loop approximation (dotted, dashed, solid) as a function of the decoupling scale $\mu_{\mathrm{dec}}$. The dash-dotted curve is what results from the formula given in Ref. [10]. See Ref. [7] for details.

DRED is currently considered the appropriate regularization method for supersymmetric theories. Applied to non-SUSY theories, it leads to evanescent couplings, with their own evolution and decoupling relations. If parameters from the non-SUSY theory are to be related to SUSY parameters, the conversion relations will typically involve these evanescent couplings. Here we took these issues into account for the derivation of $\alpha_{s}\left(\mu_{\mathrm{GUT}}\right)$ from $\alpha_{s}\left(M_{Z}\right)$ at three-loop level.

\section{References}

[1] Slides: http://indico. cern. ch/contributionDisplay.py? contribId=170\&sessionId=6\&conf Id=9499

[2] G. 't Hooft and M.J. Veltman, Nucl. Phys. B 44189 (1972).

[3] S.P. Martin and M.T. Vaughn, Phys. Lett. B 318331 (1993).

[4] W. Siegel, Phys. Lett. B 84193 (1979).

[5] R. Harlander, P. Kant, L. Mihaila, M. Steinhauser, JHEP 09053 (2006).

[6] R.V. Harlander, D.R.T. Jones, P. Kant, L. Mihaila, M. Steinhauser, JHEP 0612024 (2006).

[7] R.V. Harlander, L. Mihaila, M. Steinhauser, arXiv:0706.2953 [hep-ph].

[8] I. Jack, D.R.T. Jones, K.L. Roberts, Z. Phys. C 63151 (1994).

[9] R. Harlander, L. Mihaila, M. Steinhauser, Phys. Rev. D 72095009 (2005).

[10] J.A. Aguilar-Saavedra et al., Eur. Phys. J. C 4643 (2006).

[11] S. Bethke, Prog. Part. Nucl. Phys. 58351 (2007). 